\title{
Treatment Approaches Based on Neuroscientific Data in Patients With Obsessive-Compulsive Disorder
}

\author{
Mohammad Ansari As $1^{(\mathbb{D}}$, Parviz Asgari $^{2^{*}}{ }^{(\mathbb{D}}$, Seyedeh Zahra Bakhti ${ }^{(\mathbb{D}}$ \\ 'Department of Psychology, Andimeshk Branch, Islamic Azad University, Andimeshk, Iran \\ ${ }^{2}$ Department of Psychology, Ahvaz Branch, Islamic Azad University, Ahvaz, Iran \\ 'Department of Biology, Faculty of Sciences, University of Mohaghegh Ardabili, Ardabil, Iran
}

\begin{abstract}
Background: Obsessive-compulsive disorder $(\mathrm{OCD})$ is a severe psychological disorder that can be very disabling. Recent increasing neuroscientific data about OCD has provided important evidence that has started to change our knowledge about treatment. This paper offers an applied perspective of neuroscience on diagnosis and treatment preparation for people with OCD disease. The main aim is to enhance clinician-researcher communication to promote the conversion of developments in neuroscience research into better treatment for patients with OCD.

Methods: A PubMed search was performed using the keywords "obsessive-compulsive disorder", "neuroimaging methods", "neuroscience", and "neurological treatment". The search provided sufficient articles published in peer-reviewed journals from 1995 to 2021. The data were obtained from these various sources and were included in this review.

Results: The advancement of neurotherapeutic techniques targeting the cortico-striatal-thalamocortical (CSTC) circuit is the subject of a circuit dysregulation system. Also, according to neuroimaging studies, reductions in D2 receptors (about $-18 \%$ ), GABAA receptors $(-15 \%)$, and cingulate $5-\mathrm{HT}_{1 \mathrm{~A}}$ receptors $(-18 \%)$ in patients with OCD were reported compared with healthy controls.

Conclusion: The result suggests that DA, 5-HT, and GABA have considerable roles in anxiety- and compulsion-related disorders such as OCD. For patients with OCD, these core neuroscience themes collectively guide formulation and clinical preparation.

Keywords: Obsessive-compulsive disorder; OCD; Neuroscience; Neuro-treatment; CSTC loops; Neuroimaging.
\end{abstract}

Citation: Ansari AsI M, Asgari P, Bakhti SZ. Treatment approaches based on neuroscientific data in patients with obsessive-compulsive disorder. Clin Neurosci J. 2021;8(3):107-117. doi:10.34172/icnj.2021.24.

\section{*Correspondence to \\ Parviz Asgari, \\ PhD; Associate Professor, Department of Psychology, Ahvaz Branch, Islamic Azad University, Ahvaz, Iran Email: askary47@yahoo.com}

Published online June 30, 2021

\section{Introduction}

Sudden and intrusive thoughts and insupportable behaviors routinely happen for everybody. Similar to any category, higher levels of these desires and thoughts can exceed the standard level and disrupt a person's ordinary life. Obsessive-compulsive disorder (OCD) is a neurological disorder in which a person experiences repetitive thought (called "obsessions") or feels compelled to replicate such routines (called "compulsions") to the point of anxiety or impairment of general functioning. Over time, the person cannot manage his or her thoughts or actions. ${ }^{1}$ Hand washing, counting items, and testing to see if a door is locked are all common compulsions. These tasks occur to the point that they adversely influence the person's ordinary activities, often taking more than an hour a day. Most adults are aware that their actions are illogical. Tics, paranoia, and a higher risk of suicide are also linked to the disease. If an individual shows compulsion and obsession, the person will be diagnosed with $\mathrm{OCD}^{2}$ (Figure 1).

The prevalence of OCD is at a high level and affects approximately $3 \%$ of the population., ${ }^{3,4}$ On the other hand, its economic and socio-individual health burdens are significant for the individual and consequently for the society. An abnormal fear of contamination, uncontrollable anxiety about it, as well as excessive and frequent washing are the apparent symptoms of OCD. ${ }^{5}$ Moreover, persistence and resistance to strange behaviors are typical features of a patient with OCD. The sufferer's gap between mental purpose and behavior is termed "egodystonia" and is one of the thousands of unsolved issues still unknown to the neuroscientist. The field in the cognitive neuroscience of mental health would undoubtedly progress if any of these problems and puzzles can be solved (OCD). The age at which OCD manifests itself is bimodal, peaking in early adolescence and late childhood or again in 20-29 years. ${ }^{6}$

While treatment significantly impacts symptom 


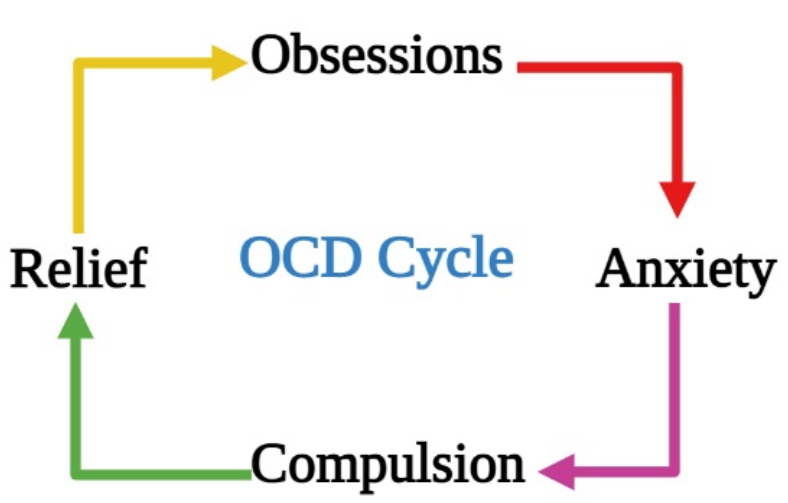

Figure 1. OCD Cycle.

progression, most patients have continuous symptoms, with up to one-fourth having a waning and waxing pattern.? The living standards of patients with OCD are declining, as are those with schizophrenia. ${ }^{8}$ Because the symptoms of OCD start from internal factors and thoughts, the patient is reluctant to retell or share his or her problem out of shame or denial. As a result, it takes almost eight years from the onset of OCD symptoms to the first pharmacological therapy. ${ }^{9}$ It was previously assumed that OCD did not respond to any treatment such as medication, systematic desensitization, or traditional dynamic psychotherapy. ${ }^{10}$ Meyer indicated that symptoms of patients with OCD increased as they were subjected to feared stimuli while refraining from undertaking compulsions, and it was the first significant nonpharmacological advance in OCD therapy. ${ }^{11}$ The effectiveness of this method, known as exposure and response prevention (ERP), was promising in hospitalized patients or outpatients. However, the significant improvement in patients did not last for more than two years after treatment. ${ }^{12}$ Although OCD has long been recognized, successful therapeutic strategies have only been discovered in the past few decades. The first randomized clinical trial of clomipramine in OCD took place in $1980 .{ }^{13}$ With the advent of selective serotonin reuptake inhibitors (SSRIs) and ERP therapy, we have undergone a time of rapid progress in the pharmacological and psychotherapeutic treatment of OCD.

Along with these biomedical breakthroughs, advancements in neuroimaging have elucidated the neural architecture that underpins OCD, opening the way for cutting-edge circuit-based neuro therapies. While these advancements have considerably changed the treatment of patients with OCD, many still suffer from significant residual symptoms. Current cognitive and therapeutic approaches to OCD mainly focus on neuroscience and biological abnormalities. In this review article, considering these approaches, modern treatment methods and their effectiveness in improving the symptoms of patients with OCD will be discussed.

\section{OCD Symptoms}

In general, $\mathrm{OCD}$, as mentioned earlier, occurs in the form of compulsive and obsessive behaviors that are performed to reduce the anxiety caused by the influx of intrusive and unconscious thoughts by the individual. ${ }^{14}$ The most typical symptoms of OCD include excessive worry about contamination and consequent constant washing. In addition, worrying about harm to self and others and as a result repeated checking is another sign of OCD. Moreover, other subtypes have been identified, including a set of symptoms related to symmetry, ritual order, and cluster focused on hoarding through factor analysis. However, OCDs are diverse and include musical, physical, religious, and sexual subtypes. ${ }^{14}$ The variety of OCD symptoms is almost constant over time and place, so that over the years and in different cultures and places, the symptoms are almost the same. In addition, the incidence and type of symptoms do not vary between adults and children, although the symptoms may change over time in each person. ${ }^{15}$

Nevertheless, the symptoms are different in patients with and without tic. ${ }^{16}$ This difference can be justified based on psychological differences. While most patients understand that their symptoms are excessive, their intuition varies, and others are labeled as having low insight. Frontal lesions can be linked to a lack of understanding of OCD signs. ${ }^{17}$ Patients with obsessional slowness may have a different form of OCD with further neurological disability. Understanding these differences and variations in people with OCD, especially from the physiological and neuroscientific aspects, can lead to more accurate diagnoses and better treatment options ${ }^{17,18}$ (Table 1).

\section{The Main Ways for OCD Diagnosis}

General medical disorders or the effects of a substance on the body that affects a person's behavior cannot be identified as an OCD by the criteria set by the Diagnostic and Statistical Manual of Mental Disorders (DSM-IV), which sets the standard criteria for identifying mental illness. $^{21}$ Various neurological lesions in the corticostriatal-thalamic-cortical pathways have been linked to obsessive-compulsive (OC) symptoms. They can occur following the application of dopamine agonists (such as methylphenidate or cocaine) or after streptococcal infection $^{22,23}$ (presumably on an autoimmune basis). ${ }^{24}$ OCD signs must be compounded by severe anxiety to be clinically meaningful. Subclinical OCD signs are typical and may appear at any time during a child's growth. Patients with OCD, on the other hand, may suffer significant consequences, including a lower standard of life. ${ }^{25,26}$ One of the most common mistakes in recognizing OCD is compulsion and obsession as inflexible personality traits in OCD classification. Although it is difficult to distinguish between axis I disorders, including 
Table 1. Symptoms of OCD

\begin{tabular}{|c|c|c|c|c|}
\hline Symptoms & Explanation & Themes & Examples & Ref. \\
\hline Obsession & $\begin{array}{l}\text { Thoughts, impulses, or images that are } \\
\text { distracting and cause anxiety or fear, are } \\
\text { repetitive, constant, and unwelcome. }\end{array}$ & $\begin{array}{l}\text { - } \quad \text { Contamination of soil phobia } \\
\text { Doubting yourself and finding it } \\
\text { impossible to accept confusion } \\
\text { Desiring things to be } \\
\text { symmetrical and tidy } \\
\text { - Thoughts of losing control and } \\
\text { hurting yourself or others } \\
\text { - Unwelcome thoughts }\end{array}$ & $\begin{array}{l}\text { Fear of being infected } \\
\text { by touching items that } \\
\text { others have touched } \\
\text { - When things are not } \\
\text { in place or facing } \\
\text { the same direction, it } \\
\text { causes much tension. }\end{array}$ & 19 \\
\hline Compulsion & $\begin{array}{l}\text { Compulsions are repeated activities that you } \\
\text { feel compelled to do in people with OCD. These } \\
\text { habits or behavioral actions are intended to } \\
\text { alleviate fear caused by the obsessions or avoid } \\
\text { anything unpleasant. Compulsions, on the other } \\
\text { hand, provide little gratification and can only } \\
\text { provide brief relief from anxiety. }\end{array}$ & $\begin{array}{ll}\text { - } & \text { Washing and cleaning } \\
\text { - } & \text { Checking } \\
\text { - } & \text { Counting } \\
& \text { Orderliness }\end{array}$ & $\begin{array}{l}\text { - Hand-washing until } \\
\text { the skin is red and } \\
\text { irritated } \\
\text { - Checking doors } \\
\text { repeatedly to make } \\
\text { sure they are locked }\end{array}$ & 20 \\
\hline
\end{tabular}

the personality traits of a person with OCD, and axis II disorders, such as OC personality disorder, there is a difference between OCD and perfectionist behaviors or extreme conscientiousness. Some symptoms such as obsession and compulsion are inseparable symptoms of other disorders such as autism, forehead lip lesions, and Tourette's syndrome.

In contrast, some disorders are easily distinguished from OCD, which can be referred to as patients with body dysmorphic ${ }^{27}$ disorder who are concerned about imagined ugliness and hypochondriasis (extreme concern about getting various diseases). These disorders fall into the spectrum of OCD while they are different from OCD. ${ }^{27}$

\section{Epidemiology of OCD}

In the United States, OCD affects $2 \%-3 \%$ of people at some point in their lives. OCD manifests itself in a bimodal fashion, with onsets in infancy and late adolescence/early adulthood. Just $15 \%$ of cases begin after 35, and twothirds begin before the age of 25. A third of all cases begin in childhood or early adolescence. Men have an early onset and a more malignant prognosis compared with women. These symptoms will last for years, and affected individuals often suffer in silence until the symptoms appear. The disorder progresses in a chronic waxing and fading pattern, with $15 \%$ of patients experiencing regression and $5 \%$ experiencing episodes of inter-episode rehabilitation..$^{28}$

\section{Etiology of OCD}

It is unclear if different forms of OC symptoms have a hereditary or environmental cause. For example, whether hoarding is etiologically linked to prototypic OC symptoms like washing, testing, and obsessing is unclear. ${ }^{29}$ It is still unclear if specific OC signs are etiologically linked to an overall propensity to suffer emotional distress. In any case, the etiology of OCD is classified into the following items:

(A) Familial/Genetic factors: Monozygotic twins have higher concordance frequencies than dizygotic twins, according to twin investigations. The incidence of OCD is higher if one of the first relatives is infected with OCD or Tourette's syndrome. In addition, if a family member has Tourette syndrome, they are more likely to develop both OCD and Tourette syndrome. Therefore, it is thought that in these families, OCD and Tourette syndrome are different expressions of the same genetic defect in the family that is inherited.

(B) Behavioral factors: Obsessions are the product of combining mental stimulation with anxiety-provoking emotions in a two-stage classical instrumental conditioning model of OCD. Compulsions are neutral habits that have been linked to reduced fear and thereby strengthened. ${ }^{30}$

(C) Neurobiological factors: Hyperactivity in frontalsubcortical thalamic pathways is implicated in the pathogenesis of OCD, according to data from imaging, pharmacological, and behavioral research (Figure 2). ${ }^{31}$ Based on this hypothesis, hyperactivity in these pathways causes excessive activity in the frontal-subcortical networks, resulting in the behavioral dysfunction seen in OCD. Increased perfusion and metabolism in the orbital frontal cortex, anterior cingulated gyrus, and head of the caudate nucleus have been found in patients with active OCD according to several pieces of functional imaging studies such as functional magnetic resonance imaging (fMRI), Single-photon emission computed tomography (SPECT), and positron emission tomography (PET). ${ }^{32}$

\section{A Glance at Neuroimaging Findings in OCD}

Since the 1980s, neuroimaging tests have shown substantial variations between healthy controls and patients with OCD. The cortico-striatal-thalamo-cortical (CSTC) paradigm for the neurobiology of OCD was proposed 


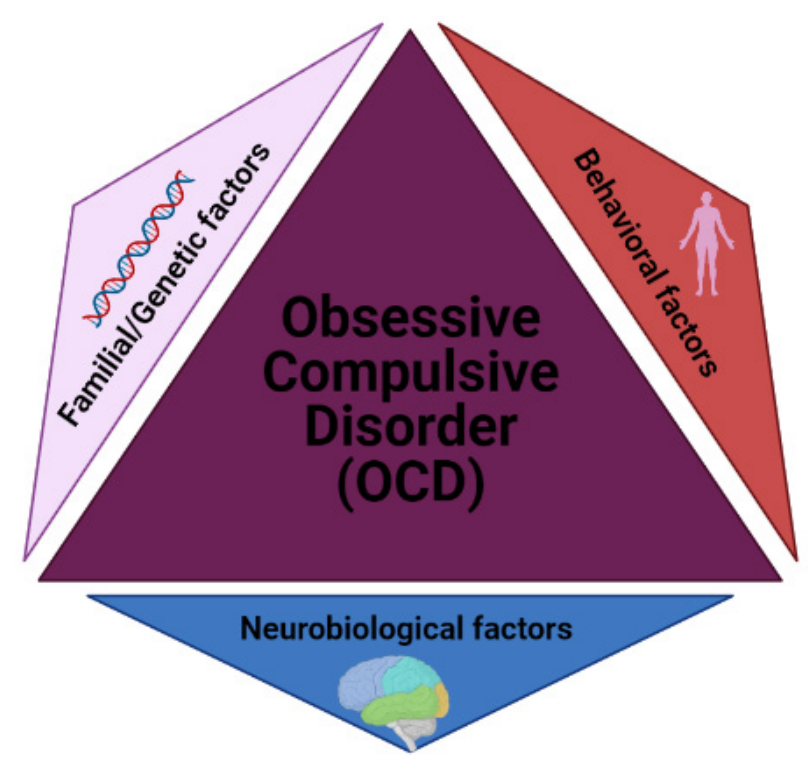

Figure 2. Risk Factors Involved in OCD Incidence.

after initial imaging studies revealed hyperactivity in the prefrontal cortex (primarily the orbitofrontal cortex $[\mathrm{OFC}]$ ), anterior cingulate cortex (ACC), and caudate nucleus. Nevertheless, research from the last two decades shows that widespread associative networks, including parts of the parietal cortex, limbic areas (including the amygdala), and the cerebellum, are involved. There are numerous studies about functional imaging studies such as fMRI, PET, SPECT, and functional near-infrared spectroscopy (fNIRS). ${ }^{33}$

PET scans calculate glucose metabolic concentrations in different parts of the brain and are linked to brain function. PET experiments have demonstrated hypermetabolism of OCD in various regions of the PFC since the early 1980s. In a study by Le Jeune and colleagues, 10 patients were selected randomly and treated with high-frequency bilateral deep brain stimulation of the subthalamic nucleus (STN DBS). By PET analysis, the level of glucose metabolism in the right frontal middle gyrus was compared to the healthy group. All 10 patients had median Yale-Brown Obsessive-Compulsive Scale (Y-BOCS) ratings of 31 (minimum: 18, maximum: 36) for "Off-Stimulation" and 19 (minimum: 0, maximum: 30) for "On-Stimulation" $(P=0.05)$. Compared to the stable control group, patients with OCD displayed hyper metabolism in the right frontal middle and superior gyri, right parietal lobe, postcentral gyrus, and bilateral putamen in off-stimulation status. On-Stimulation conditions showed a substantial reduction in cortical metabolism in the left cingulate gyrus and left frontal medial gyrus compared to Off-Stimulation conditions. Furthermore, Y-BOCS score improvements during onstimulation conditions were positively associated with PET signal changes at the OFC-medial prefrontal cortex border and between PET signal changes and Y-BOCS score changes in on-stimulation status. According to this report, the therapeutic role of STN DBS seems to be linked to a reduction in prefrontal cortex metabolism, according to this report. ${ }^{34}$

Blood flow, receptor availability, and drug absorption are all investigated in SPECT experiments. Reduced uptake in the OFC, ACC, and posterior cingulate cortex (PCC), temporal, parietal, and occipital cortices and improved uptake in the cerebellum have been observed in SPECT findings in patients with OCD. ${ }^{35}$ In one study, resting regional cerebral blood flow $(\mathrm{rCBF})$ was examined in 26 untreated patients with OCD and 22 healthy control people. In the right lateral OFC and the left dorsal ACC, patients with OCD have slightly lower ECD absorption than control subjects (Z-scores greater than 3.29, $P<0.001) .{ }^{36}$

In another study, single photon emission computed tomography with hexamethyl propylenamine oxime (HMPAO SPECT) was used to test $\mathrm{rCBF}$ in 16 drug-free Brazilian patients with OCD and 17 healthy subjects. The right superior and inferior frontal cortex and the right and left thalamus were shown to have substantially higher rCBF after covariance analysis. ${ }^{37}$ Overall, in PET and SPECT studies, positively correlations have been between OFC, DLPFC, lateral and medial temporal cortex, and inferior parietal lobule uptakes concerning Y-BOCS symptom intensity. However, negative correlations have been found with posterior cingulate uptake and serotonin transporter activity in hypothalamic and thalamic regions. In previous neuroimaging studies, $5-\mathrm{HT}_{1 \mathrm{~A}}, 5-\mathrm{HT}_{2 \mathrm{~A}}, \mathrm{D} 2$, and GABA were compared between 504 patients with anxiety disorder and 593 controls via PET or SPECT methods. Significant decreases of striatal D2 receptors in OCD $(-18 \%)$, cingulate $5-\mathrm{HT}_{1 \mathrm{~A}}$ receptors $(-18 \%)$, striatal D2 receptors $(-21 \%)$ and frontal $(-14 \%)$, temporal $(-14 \%)$, occipital $(-13 \%)$ and cingulate GABAA receptors $(-15 \%)$ were reported in patients including those with OCD. Moreover, 5-HT and GABA can inhibit DAergic neurotransmission, so the reduction in $5-\mathrm{HT}_{1 \mathrm{~A}}$ and GABAA can lead to high-level activity of the mesolimbic DAergic system. Based on the results, it can be acknowledged that DA, 5-HT, and GABA have considerable role in anxiety- and compulsion-related disorders such as OCD. ${ }^{38}$

Despite the vast amount of PET and SPECT trials published, they all had flaws, such as limited sample sizes and inconsistencies in inclusion/exclusion criteria for comorbidity, symptom dimensions, and drug status. Furthermore, the SPECT experiments used different reference regions for estimation, using the cerebellum and some using the occipital cortex. Because of these limits, it is impossible to extrapolate the results. Many trials have looked at pre- and post-treatment shifts, making them a valuable method for understanding the neurobiology 
of OCD and defining treatment modulation areas. FMRI is focused on the measurement of improvements in activity and connectivity in various brain areas as a function of blood oxygen level dependency. Increased functional interaction between the caudate, putamen, and OFC and the ACC and para-hippocampal regions has been discovered in resting-state fMRI studies. Functional integration was compared in a recent metaanalysis of 18 whole-brain resting-state fMRI trials with 572 healthy controls and 541 patients. In OCD, researchers discovered reduced connectivity between the frontoparietal and salience networks, and between the salience, frontoparietal, and default mode networks, as well as general dysconnectivity (no apparent increase/ decrease in connectivity) within the default mode, and frontoparietal and salience networks. ${ }^{39}$

FNIRS is a modern, compact, and simple-to-use optical imaging modality. It tests oxygenated and deoxygenated hemoglobin levels in brain regions to assess brain function and is established based on the concept of neurovascular coupling. There are two publications based on fNIRS among 12 and 20 OCD patients. In one study, 20 patients with OCD and 20 controls were selected. The verbal fluency task consisted of a 30-second pre-task, three repetitions of a 20 -second verbal fluency task (total 60 seconds), and a 70-second post-task cycle to evoke near-infrared spectroscopic activation. In 14 channels spread around the left and right dorsolateral prefrontal cortex and frontopolar regions, Z-scores for differences in oxygenated hemoglobin concentration were matched between patients with OCD and controls. The OCD group had significantly lower oxygenated hemoglobin concentrations in the right dorsolateral prefrontal cortex than the controls. However, the differences were not statistically meaningful after several associations were corrected. For the patients with OCD, the mean \pm SD Y-BOCS score was $19.6 \pm 8.2$, and the mean \pm SD MOCI (Maudsley Obsessive Compulsive Inventory) score was $15.5 \pm 5.8$, while the mean \pm SD MOCI score for control subjects was $4.3 \pm 3.5$. There were significant differences in the mean MOCI score $(\mathrm{t}=-7.39, \mathrm{df}=38, P<0.001)$ and verbal fluency scores $(\mathrm{t}=3.892, \mathrm{df}=38, P<0.001)$ between the two groups. Their founding support of this hypothesis that the dorsolateral prefrontal cortex has an essential role in OCD pathophysiology. ${ }^{40}$

In another study, neurobiological function in 12 patients with OCD and 12 healthy subjects were analyzed by fNIRS. During the execution of a Stroop color-word task, the relative concentrations of oxyhemoglobin (oxy-Hb) were determined with prefrontal probes per 0.1 s utilizing 24-channel NIRS. The OCD group's oxy$\mathrm{Hb}$ changes in the prefrontal cortex were substantially lower than the control group, especially in the left lateral prefrontal cortex. These findings show that, as assessed by NIRS, patients with OCD have decreased prefrontal hemodynamic responses. ${ }^{41}$ According to these two studies, patients with OCD, compared to healthy subjects, have lower activation in the DLPFC during the verbal fluency test and in the left lateral PFC during the Stroop task. There are some mega-analytical studies enhancing neuroimaging and genetics by meta-analysis that gathered data from 16 countries to produce neuroimaging metaanalyses of nearly 2000 patients from all age ranges, which leads to growing our understanding of the neurobiology of OCD and its progression over time. These massive multicentric studies help overcome the limitations of current neuroimaging studies, such as limited sample sizes and discrepancies in inclusion/exclusion criteria for comorbidity, heterogeneous symptom dimensions, and drug status, and enable data comparison. ${ }^{42}$

\section{Role of Neurochemistry in OCD Development}

Considering the established efficacy of serotonin (5-HT) reuptake inhibitors, a disruption in serotonergic function is considered a primary factor or OCD in recent years. ${ }^{43}$ SSRI and clomipramine are some of these inhibitors. Neuroimaging experiments examining the density of 5-HT receptor and transporter utilizing SPECT and PET have shown mixed findings. However, they do vindicate localized 5-HT anomalies in OCD. In remarkably reduced availability of 5-HT transporter in the midbrain and thalamus, enhanced availability of $5-\mathrm{HT}_{2 \mathrm{~A}}$ receptor in the caudate, and reduced availability of $5-\mathrm{HT}_{2 \mathrm{~A}}$ receptor in the prefrontal regions are among them. ${ }^{44}$ Lower synaptic serotonin inside the CSTC circuit involved in OCD is thought to be the cause of these results (Figure 3).

Despite the mixed results of these trials and the reality that approximately half of the patients with OCD treated with an SSRI may not improve, neurotransmitter mechanisms other than 5-HT are likely to play a part in the OCD pathophysiology. ${ }^{45}$ Single-photon absorption and PET in patients with OCD showed increased binding of striatal dopamine transporter density and decreased striatal D2/D3 and D1 receptors, indicating dopaminergic hyperactivity in this area. As a result, metaanalyses support the use of antipsychotic drugs as SSRI augmentation therapies for OCD, especially the highaffinity D2 receptor antagonist risperidone. Antipsychotic augmentation, on the other hand, works on around $1 / 3$ of patients with treatment-resistant $\mathrm{OCD},{ }^{44}$ leaving physicians looking for other options. ${ }^{46,47}$

A function for impaired glutamatergic neurotransmission in the pathophysiology of OCD is approved by many pieces of evidence. ${ }^{48}$ By magnetic resonance spectroscopy, the accumulation of glutamate and its related glutamine metabolite seems to be normalized by paroxetine treatment in the caudal nucleus of children with OCD during preliminary research. ${ }^{48}$ However, recent attempts to reproduce these results have overwhelmingly failed. Numerous genes involved in 

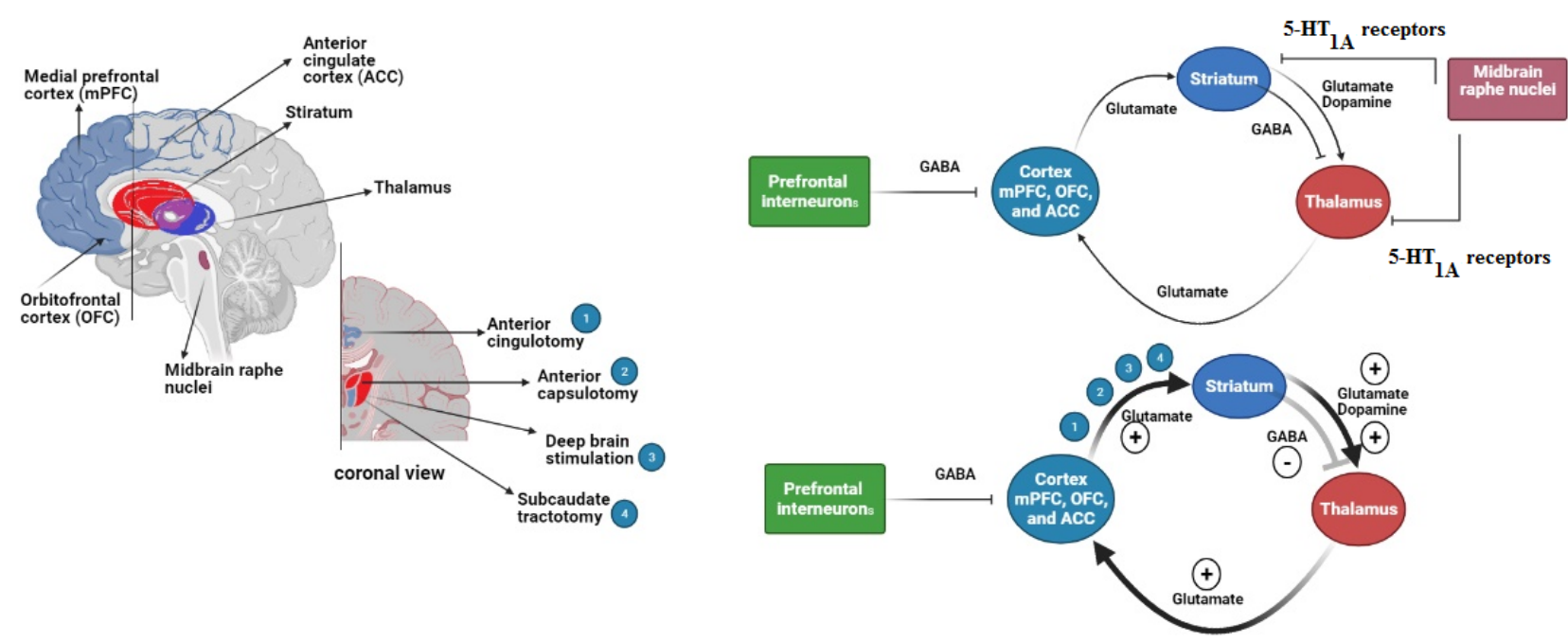

Figure 3. Inhibitory interactions and normal excitatory leading to cortico-striatal-thalamo-cortical (CSTC) circuitry are seen in uncomplicated manner. Midbrain raphe nuclei and mPFC interneurons both show decreased serotonergic and GABAergic inhibitory tone in the hyper activation of CSTC circuit involved in OCD, resulting in striatal dopaminergic and glutamatergic hyperactivity driving unrestrained CSTC activity. Neurosurgical interventions that change the hyperactive CSTC circuitry, have been shown to help people with serious, treatmentresistant OCD. Subcaudate tractotomy, anterior capsulotomy, and anterior cingulotomy are surgical lesions that may be done with an open or gamma knife approach. Deep brain stimulation is done only posterior to the capsulotomy lesion target, in the ventral striatum and ventral capsule.

glutamate expression and metabolism, have been linked to $\mathrm{OCD}$, prompting the development of transgenic animal models of the disorder. Several minor preclinical studies have looked at glutamate modulating interventions such as memantine, $\mathrm{N}$-acetylcysteine, and riluzole as possible SSRI augmentation therapies for OCD. At least in a subset of patients with OCD, this shows positive outcomes. ${ }^{49} \mathrm{In}$ a limited placebo-controlled crossover study, ketamine decreased OCD symptoms after a single intravenous injection. However, there is little gain in an open-label trial for patients who are more treatment-resistant and show severe symptoms of OCD. Overall, further research is required to decide if glutamatergic drugs should be used to treat OCD. If glutamic drugs are helpful, it should be considered in which group of patients it will be most effective. $^{50}$

Any disruption in the function of the GABA in OCD is very important. In patients with OCD, however, anomalies in prefrontal inhibitory neurotransmission have been discovered, which are thought to be caused by low GABAergic activation and low medial prefrontal and orbitofrontal cortical GABA levels. Furthermore, an abrupt rise in GABA levels in the medial prefrontal cortex following ketamine infusion was linked to a dramatic decrease in OCD symptoms. These results highlight the need for further research into the abnormalities of the GABAergic role in OCD. ${ }^{44,51}$

The results indicate that a variety of neurochemical anomalies could cause OCD. Overactive CSTC circuits, as shown by glutamatergic hyperactivity and striatal dopaminergic neurons, seem to be at the core of OCD. ${ }^{52}$
The cause of this hyperactive condition is unknown; however, it may be caused by primary defects in the gene involved in glutamate metabolism found in CSTC brain regions, or it may be secondary to decreased midbrain and prefrontal serotonergic function. ${ }^{53}$ Via activation of $5-\mathrm{HT}_{1 \mathrm{~A}}$ receptors, serotonergic projections from the raphe nuclei suppress dopaminergic activity in the striatum and thalamic activity, while stimulatory $5-\mathrm{HT}_{2 \mathrm{~A}}$ autoreceptors on prefrontal GABA interneurons inhibit striatal glutamatergic activity. ${ }^{54,55}$ As a result, SSRIs can help alleviate symptoms of OCD via moderating glutamatergic hyperactivity and striatal dopaminergic neurons, through increased serotonergic inhibition (Figure 3). Reduction in the level of prefrontal GABAergic inhibition can enhance striatal activation in addition to serotonergic deficits, either directly or indirectly through projections to the OFC. Although this paradigm is simplistic, it serves as a starting point for more research into the pathophysiology of OCD and the advancement of new therapeutic methods. $^{51}$

OCD neurobiology is linked to the ACC. Few trials, however, have looked directly at functional and neurochemical anomalies in the rostral subdivision of the ACC (rACC) in OCD patients. In a study by Brennan et al, the role and neurochemistry of the rACC in people with OCD and people without OCD using fMRI during an emotional counting Stroop task and single-voxel J-resolved proton magnetic resonance spectroscopy (1H-MRS) in the rACC was compared. Linear regression was used to investigate inter-group variations in rACC activation and glutamine/glutamate ratio (Gln/Glu), Glu, 
and Gln levels and correlations between rACC activation, Gln/Glu, Glu, Gln, behavioral, and clinical measures. However, there was no difference in Gln/Glu, Glu, or Gln in the rACC between classes, and there was no connection between reduced rACC deactivation and Gln/Glu, Glu, or Gln in the OCD community. These results support rACC dysfunction as a cause of OCD, but they contradict an underlying connection to irregular rACC glutamatergic neurotransmission. ${ }^{56}$

Although much progress has been made in understanding the social, neurological, and neurochemical aspects of OCD individually, their interplay, especially during psychotherapy, remains a mystery. In one study, seventeen participants were tested before and during inpatient psychotherapy at all three stages using psychiatric questionnaires, fMRI, and venipuncture. At similar time intervals, seventeen controllers were scanned. First, improvements in symptom severity, whole-brain and regional function, cortisol, serotonin, dopamine, brain-derived neurotrophic factor, and immunological parameters (IL-6, IL-10, TNF) were examined independently and aftercare. Second, stepwise linear simulation was used to discover relationships between the level variables. Since psychotherapy, the severity of OC, suicidal, and general symptoms was substantially decreased. The ACC activation, frontal areas, precuneus, and putamen had all diminished dramatically on a neuronal basis. On a neurochemical basis, no significant improvements were discovered. When the levels were associated, a critical model was discovered that explained the decrease in putamen neural activity by rising cortisol, IL-6, and dopamine concentrations. ${ }^{57}$

While some studies highlight the role of neurochemical agents in developing OCD symptoms, others do not consider their role to be significant. However, according to the available evidence, more extensive studies are needed to clarify the role of neurochemical agents and their use in the treatment of OCD.

\section{Neurotherapeutics Approaches in OCD}

Themostwell-acceptedbrain circuitmodelofpsychological disorders is OCD, based on the concept of compulsions as habitual, almost unconscious behavior. Most of the habits are related to repetitive circuits called CSTC loops that link the cerebral cortex to the basal ganglia. ${ }^{58}$ CSTC loops will be hyperconnected or hyperactive and self-exciting in an uncontrolled positive feedback loop in the CSTC model. This increases the need to execute compulsions, which in turn strengthens/consolidates the practice of executing compulsions. Patients who use exposure and reaction avoidance treatment instead of practicing not to act on their compulsions will be able to intensify the chain. ${ }^{59}$ Obsessions or compulsions: who comes first, obsessions or compulsions?

The definition of CSTC circuit(s) has changed over time. Models have been based on an indirect in contrast to a direct pathway, with OCD being a misalignment of their relative drive. Although primary models assumed multiple parallel CSTC loops, and each loop was connected to different sides of basal ganglia and cortex, the advanced models have shown cascade spiral connections across the CSTC loops. However, according to all model variants, CSTC models should be hyperconnected and hyperactive in OCD. ${ }^{32}$ Human and animal trials support the model of the CSTC structure. The dorsal ACC, OFC, and caudate nucleus, which are involved in these circuits, are abnormally active in individuals with OCD at rest and with symptom arousal. This hyperactivity decreases with behavioral and pharmacological care. ${ }^{60}$ More recent research has applied this to physical connectivity as measured by fMRI and has discovered anomalies in various connections within the CSTC circuits once again. ${ }^{61}$ Executive function activities that specifically rely on these circuits indicate anomalies in patients with OCD. ${ }^{62}$

At last, SAPAP3, a crucial glutamate gene for CSTC loop production, has been knocked out in mice. ${ }^{63}$ Such mice have irregular cortico-striatal synaptic properties as well as abnormal (compulsive) grooming. Grooming ceases until the CSTC abnormality is reversed; pathological grooming occurs when nongenetic factors cause it. Although the mice model of compulsive grooming is a straightforward model for humans OCD, in general, its result supports the notion that CSTC defects play a critical role in OCD. ${ }^{64}$

The current neurocircuit theory has affected neurotherapeutic methods, such as efforts to treat OCD by intervening specifically with the function of the CSTC loop. Ablative surgeries and brain stimulation are included, with the latter categorized as invasive (deep brain stimulation) and non-invasive (transcranial magnetic stimulation). Subcaudate tractotomy, anterior capsulotomy, limbic leucotomy, and anterior cingulotomy are some of the surgical options. Both are backed by free-label data, which show response rates in the $36-75 \%$ (defined as a $36 \%$ or tremendous increase on the YBOCS) and overall favorable adverse effect profiles in terms of mortality or long-term morbidity. ${ }^{65}$ The value of response rate varies more between trials of the same protocol than between procedures. Capsulotomy, unlike the others, has been transformed into a gamma knife surgical technique, allowing for a simple sham-controlled experiment. ${ }^{66}$ Although the trial's primary analysis did not achieve statistical significance, long-term follow-up revealed improvements similar to open capsulotomy.

With the chance of adapting stimulation dose in response to patient improvement, brain stimulation extends the circuit-disrupting positions of the lesions. One of the most important measures is magnetic stimulation of the transcranial area, which quickly leads to depressive symptoms in patients. ${ }^{67}$ The left dorsolateral 
prefrontal cortex target, which is used to treat depression, has little effect on OCD. The targeting supplementary motor area, which is more nearly connected to the c CSTC loops, caused modest positive effects in two experiments, including a meta-analysis study and two sham-controlled transcranial magnetic stimulations. ${ }^{68}$ The supplementary motor area, while it is sometimes deemed experimental by insurers, transcranial magnetic stimulation can be recommended as an alternative to ERP therapy and evidence-based medicine. ${ }^{69}$ Stimulation of the brain deeply in the clinical challenge, the treatment chosen by the patient, is a more invasive procedure in which electrodes are implanted directly into the CSTC loop systems to provide sustained high-frequency stimulation. Some trials in Europe indicated that limbic regions in the subthalamic nucleus are an effective DBS site. Nevertheless, the ventral internal capsule is more interesting for most of the research teams. ${ }^{70}$

The DBS site, a white matter center with Many CSTC loops, has randomized and free-label clinical trial results to back up its effectiveness in OCD, with response rates ranging from $33-75 \% .{ }^{71,72}$ An internal capsule of the ventral striatum based on those results, the US FDA approved DBS for OCD under a Humanitarian Device Exemption. DBS has many advantages over ablative treatments, including the fact that it is reversible and flexible. It enables tests of the brain with and without stimuli, confirming that DBS alters the function of various CSTC systems. ${ }^{72,73}$ On the other hand, those same properties cause patients to rebound as a result of system failures and possible stimulus overdoses, resulting in hypomania. ${ }^{74,75}$ Closed-loop systems that self-adjust stimulus in response to CSTC loop operation could fix these issues, but they are still a work in progress. ${ }^{74,76}$ Both genetic and circuit research have traditionally viewed OCD as a discrete condition, a significant caveat. Many phenotypic subtypes of OCD have been identified by psychological factor studies, each with its biology; the same can be said for the occurrence or lack of comorbid tics. Experimental and clinical psychology are driving to a dimensional definition of disease, identifying patients' impairments in particular areas of functioning, as previously stated. Since multiple CSTC loops are thought to lead to various aspects of the condition, OCD may be more amenable to the definition. If an accurate, quantifiable dimensional structure can be established, OCD neurotherapeutics' targeting can be improved. ${ }^{76}$

\section{Conclusion}

For the past 30 years, progress in discovering the molecular, neurological, and developmental OCD underpinnings has surpassed that of any other medical condition. We anticipate that many core fields of OCD science will experience rapid growth and advancement in the future. First, because of the significant phenotypic variability in the diagnosis of OCD, further research into the biological discrepancies between subtypes of OCD symptoms should be conducted using deep phenotyping, a Research Domain Criteria (RDoC) methodology, and sophisticated neuroimaging techniques. In the next step, given the increasing body of evidence that glutamatergic dysfunction plays a role in OCD, new glutamatemodulating therapies in OCD are warranted. Then, considering the proof of extinction learning deficits in OCD, more research into alternative pharmacologic, neuro-modulatory, or behavioral interventions to supplement ERP therapy should be prioritized. Moreover, there is much need for the development and refinement of neurotherapeutic/neurostimulation treatments for OCD. Finally, progress in these fields holds promise for medical approaches that can be administered neuro-scientifically for patients with OCD on an individualized basis using a personalized medicine approach to improve outcomes.

While a neurocircuit-based taxonomy for OCD is still in its beginnings, information gained over the last few decades about various neurocognitive functions related to OCD and their underlying neural pathways may offer insights into possible disease mechanisms and novel neurocircuitbased treatment goals. Given that the standard medical method to identify psychiatric illness based on clusters of symptoms fails to show consistent and accurate circuitry defects across persons diagnosed with the same condition, medications that target particular circuits allow for treating their underlying symptomatology rather than the disorder as a whole. If specific changes in circuit regulating functions such as anxiety, subjective sensory perceptions, repetitive habits, reaction inhibition, reward, and executive function identify specific clinical profiles of OCD, treatments addressing those circuits and clinical profiles may be created. We hypothesize that effective management of OCD would necessitate treating different circuits, depending on the clinical profile, since OCD phenotypes include conflicting circuits. Given that some of these clinical profiles are used in other conditions (for example, sensory phenomena in tic disorders), specific circuit-based therapy methods may be used to address these clinical profiles through diseases rather than inside a particular categorical diagnosis until they are well defined.

\section{Author's contribution}

M.A and Z.B. contributed to the collecting data and the writing of the manuscript. P.A. and contributed to revision and supervised the project.

\section{Conflict of Interest Disclosures}

The authors do not have any conflict of interest.

Ethical Statement

Not applicable. 


\section{Funding}

This paper was not funded.

\section{References}

1. Park NY. OCD and errors in financial decisions. Econ Bull. 2018;38(4):1970-7.

2. Angelakis I, Gooding P, Tarrier N, Panagioti M. Suicidality in obsessive compulsive disorder (OCD): a systematic review and meta-analysis. Clin Psychol Rev. 2015;39:1-15. doi: 10.1016/j.cpr.2015.03.002.

3. Stein DJ, Costa DLC, Lochner C, Miguel EC, Reddy YCJ, Shavitt RG, et al. Obsessive-compulsive disorder. Nat Rev Dis Primers. 2019;5(1):52. doi: 10.1038/s41572-019-01023.

4. Shephard E, Stern ER, van den Heuvel OA, Costa DLC, Batistuzzo MC, Godoy PBG, et al. Toward a neurocircuitbased taxonomy to guide treatment of obsessivecompulsive disorder. Mol Psychiatry. 2021. doi: 10.1038/ s41380-020-01007-8.

5. Ruscio AM, Stein DJ, Chiu WT, Kessler RC. The epidemiology of obsessive-compulsive disorder in the National Comorbidity Survey Replication. Mol Psychiatry. 2010;15(1):53-63. doi: 10.1038/mp.2008.94.

6. Anholt GE, Aderka IM, van Balkom AJ, Smit JH, Schruers K, van der Wee NJ, et al. Age of onset in obsessive-compulsive disorder: admixture analysis with a large sample. Psychol Med. 2014;44(1):185-94. doi: 10.1017/s0033291713000470.

7. Pinto A, Mancebo MC, Eisen JL, Pagano ME, Rasmussen SA. The Brown Longitudinal Obsessive Compulsive Study: clinical features and symptoms of the sample at intake. J Clin Psychiatry. 2006;67(5):703-11. doi: 10.4088/jcp. v67n0503.

8. Subramaniam M, Soh P, Vaingankar JA, Picco L, Chong SA. Quality of life in obsessive-compulsive disorder: impact of the disorder and of treatment. CNS Drugs. 2013;27(5):36783. doi: 10.1007/s40263-013-0056-z.

9. Ramos-Cerqueira AT, Torres AR, Torresan RC, Negreiros AP, Vitorino CN. Emotional burden in caregivers of patients with obsessive-compulsive disorder. Depress Anxiety. 2008;25(12):1020-7. doi: 10.1002/da.20431.

10. Foa EB, Yadin E, Lichner TK. Exposure and Response (Ritual) Prevention for Obsessive Compulsive Disorder: Therapist Guide. Oxford University Press; 2012.

11. Meyer V. Modification of expectations in cases with obsessional rituals. Behav Res Ther. 1966;4(4):273-80. doi: 10.1016/0005-7967(66)90023-4.

12. Foa EB, Goldstein A. Continuous exposure and complete response prevention in the treatment of obsessivecompulsive neurosis. Behav Ther. 1978;9(5):821-9. doi: 10.1016/s0005-7894(78)80013-6.

13. Pittenger C, Kelmendi B, Bloch M, Krystal JH, Coric V. Clinical treatment of obsessive compulsive disorder. Psychiatry (Edgmont). 2005;2(11):34-43.

14. Stein DJ, Rapoport JL. Cross-cultural studies and obsessivecompulsive disorder. CNS Spectr. 1996;1(1):42-6. doi: $10.1017 /$ S1092852900000675.

15. Swedo SE, Pietrini P, Leonard HL, Schapiro MB, Rettew DC, Goldberger EL, et al. Cerebral glucose metabolism in childhood-onset obsessive-compulsive disorder. Revisualization during pharmacotherapy. Arch Gen Psychiatry. 1992;49(9):690-4. doi: 10.1001/ archpsyc.1992.01820090018003.

16. Miguel EC, do Rosário-Campos MC, Shavitt RG, Hounie AG, Mercadante MT. The tic-related obsessive-compulsive disorder phenotype and treatment implications. Adv Neurol. 2001;85:43-55.

17. Ames D, Cummings JL, Wirshing WC, Quinn B, Mahler M. Repetitive and compulsive behavior in frontal lobe degenerations. J Neuropsychiatry Clin Neurosci. 1994;6(2):100-13. doi: 10.1176/jnp.6.2.100.

18. Gholampour S, Bahmani M. Hydrodynamic comparison of shunt and endoscopic third ventriculostomy in adult hydrocephalus using in vitro models and fluidstructure interaction simulation. Comput Methods Programs Biomed. 2021;204:106049. doi: 10.1016/j. cmpb.2021.106049.

19. Amir N, Freshman M, Ramsey B, Neary E, Brigidi B. Thought-action fusion in individuals with OCD symptoms. Behav Res Ther. 2001;39(7):765-76. doi: 10.1016/s00057967(00)00056-5.

20. Nedeljkovic M, Moulding R, Kyrios M, Doron G. The relationship of cognitive confidence to OCD symptoms. J Anxiety Disord. 2009;23(4):463-8. doi: 10.1016/j. janxdis.2008.10.001.

21. Spitzer RL, Wakefield JC. DSM-IV diagnostic criterion for clinical significance: does it help solve the false positives problem? Am J Psychiatry. 1999;156(12):1856-64. doi: 10.1176/ajp.156.12.1856.

22. Hashemi Goradel N, Baker AT, Arashkia A, Ebrahimi N, Ghorghanlu S, Negahdari B. Oncolytic virotherapy: challenges and solutions. Curr Probl Cancer. 2021;45(1):100639. doi: 10.1016/j. currproblcancer.2020.100639.

23. Ebrahimi N, Amirmahani F, Sadeghi B, Ghanaatian M. Trichoderma longibrachiatum derived metabolite as a potential source of anti-breast-cancer agent. Biologia. 2021;76(5):1595-601. doi: 10.1007/s11756-021-00705-0.

24. Stasik SM, Naragon-Gainey K, Chmielewski M, Watson D. Core OCD symptoms: exploration of specificity and relations with psychopathology. J Anxiety Disord. 2012;26(8):859-70. doi: 10.1016/j.janxdis.2012.07.007.

25. Stein DJ, Allen A, Bobes J, Eisen JL, Figuera ML, Iikura Y, et al. Quality of life in obsessive-compulsive disorder. CNS Spectr. 2000;5(S4):37-9. doi: 10.1017/s1092852900025025.

26. Ghanaat M, Hashemi Goradel N, Arashkia A, Ebrahimi N, Ghorghanlu S, Veisi Malekshahi Z, et al. Virus against virus: strategies for using adenovirus vectors in the treatment of HPV-induced cervical cancer. Acta Pharmacol Sin. 2021. doi: 10.1038/s41401-021-00616-5.

27. Goodman WK. Obsessive-compulsive disorder: diagnosis and treatment. J Clin Psychiatry. 1999;60 Suppl 18:27-32.

28. Nazeer A, Latif F, Mondal A, Azeem MW, Greydanus DE. Obsessive-compulsive disorder in children and adolescents: epidemiology, diagnosis and management. Transl Pediatr. 2020;9(Suppl 1):S76-S93. doi: 10.21037/tp.2019.10.02.

29. Rajendram R, Kronenberg S, Burton CL, Arnold PD. Glutamate genetics in obsessive-compulsive disorder: a review. J Can Acad Child Adolesc Psychiatry. 
2017;26(3):205-13.

30. Bozorgmehr A, Ghadirivasfi M, Shahsavand Ananloo E. Obsessive-compulsive disorder, which genes? which functions? which pathways? an integrated holistic view regarding OCD and its complex genetic etiology. J Neurogenet. 2017;31(3):153-60. doi: 10.1080/01677063.2017.1336236.

31. Shoja Shafti S. Obsessive-compulsive traits, as behavioral invention \& foundation of cultural-historical evolution: a pilot study. Am J Biomed Sci Res. 2019;3(3):201-5. doi: 10.34297/ajbsr.2019.03.000662.

32. Pauls DL, Abramovitch A, Rauch SL, Geller DA. Obsessive-compulsive disorder: an integrative genetic and neurobiological perspective. Nat Rev Neurosci. 2014;15(6):410-24. doi: 10.1038/nrn3746.

33. Hazari N, Narayanaswamy JC, Venkatasubramanian G. Neuroimaging findings in obsessive-compulsive disorder: a narrative review to elucidate neurobiological underpinnings. Indian J Psychiatry. 2019;61(Suppl 1):S9S29. doi: 10.4103/psychiatry.IndianJPsychiatry_525_18.

34. Le Jeune F, Vérin M, N’Diaye K, Drapier D, Leray E, Du Montcel ST, et al. Decrease of prefrontal metabolism after subthalamic stimulation in obsessive-compulsive disorder: a positron emission tomography study. Biol Psychiatry. 2010;68(11):1016-22. doi: 10.1016/j.biopsych.2010.06.033.

35. Karadağ F, Kalkan Oğuzhanoğlu N, Yüksel D, Kıraç S, Cura C, Ozdel O, et al. The comparison of preand post-treatment $(99 \mathrm{~m}) \mathrm{Tc}$ HMPAO brain SPECT images in patients with obsessive-compulsive disorder. Psychiatry Res. 2013;213(2):169-77. doi: 10.1016/j. pscychresns.2012.07.005.

36. Busatto GF, Zamignani DR, Buchpiguel CA, Garrido GE, Glabus MF, Rocha ET, et al. A voxel-based investigation of regional cerebral blood flow abnormalities in obsessivecompulsive disorder using single photon emission computed tomography (SPECT). Psychiatry Res. 2000;99(1):15-27. doi: 10.1016/s0925-4927(00)00050-0.

37. Lacerda AL, Dalgalarrondo P, Caetano D, Camargo EE, Etchebehere EC, Soares JC. Elevated thalamic and prefrontal regional cerebral blood flow in obsessivecompulsive disorder: a SPECT study. Psychiatry Res. 2003;123(2):125-34. doi: 10.1016/s0925-4927(03)00061-1.

38. Nikolaus S, Antke C, Beu M, Müller HW. Cortical GABA, striatal dopamine and midbrain serotonin as the key players in compulsive and anxiety disorders--results from in vivo imaging studies. Rev Neurosci. 2010;21(2):119-39. doi: 10.1515/revneuro.2010.21.2.119.

39. Gürsel DA, Avram M, Sorg C, Brandl F, Koch K. Frontoparietal areas link impairments of large-scale intrinsic brain networks with aberrant fronto-striatal interactions in OCD: a meta-analysis of restingstate functional connectivity. Neurosci Biobehav Rev. 2018;87:151-60. doi: 10.1016/j.neubiorev.2018.01.016.

40. Hirosawa R, Narumoto J, Sakai Y, Nishida S, Ishida T, Nakamae T, et al. Reduced dorsolateral prefrontal cortical hemodynamic response in adult obsessive-compulsive disorder as measured by near-infrared spectroscopy during the verbal fluency task. Neuropsychiatr Dis Treat. 2013;9:955-62. doi: 10.2147/ndt.s45402.
41. Okada K, Ota T, Iida J, Kishimoto N, Kishimoto T. Lower prefrontal activity in adults with obsessive-compulsive disorder as measured by near-infrared spectroscopy. Prog Neuropsychopharmacol Biol Psychiatry. 2013;43:7-13. doi: 10.1016/j.pnpbp.2012.11.013.

42. Boedhoe PSW, Schmaal L, Abe Y, Alonso P, Ameis SH, Anticevic A, et al. Cortical abnormalities associated with pediatric and adult obsessive-compulsive disorder: findings from the ENIGMA Obsessive-Compulsive Disorder Working Group. Am J Psychiatry. 2018;175(5):453-62. doi: 10.1176/appi.ajp.2017.17050485.

43. Pittenger $\mathrm{C}$, Bloch $\mathrm{MH}$. Pharmacological treatment of obsessive-compulsive disorder. Psychiatr Clin North Am. 2014;37(3):375-91. doi: 10.1016/j.psc.2014.05.006.

44. Dougherty DD, Brennan BP, Stewart SE, Wilhelm S, Widge AS, Rauch SL. Neuroscientifically informed formulation and treatment planning for patients with obsessive-compulsive disorder: a review. JAMA Psychiatry. 2018;75(10):1081-7. doi: 10.1001/jamapsychiatry.2018.0930.

45. Jenike MA. Clinical practice. Obsessive-compulsive disorder. N Engl J Med. 2004;350(3):259-65. doi: 10.1056/ NEJMcp031002.

46. Dold $M$, Aigner $M$, Lanzenberger $R$, Kasper $S$. Antipsychotic augmentation of serotonin reuptake inhibitors in treatment-resistant obsessive-compulsive disorder: a meta-analysis of double-blind, randomized, placebo-controlled trials. Int J Neuropsychopharmacol. 2013;16(3):557-74. doi: 10.1017/s1461145712000740.

47. Gholampour S, Mehrjoo S. Effect of bifurcation in the hemodynamic changes and rupture risk of small intracranial aneurysm. Neurosurg Rev. 2021;44(3):170312. doi: 10.1007/s10143-020-01367-3.

48. Moore GJ, MacMaster FP, Stewart C, Rosenberg DR. Case study: caudate glutamatergic changes with paroxetine therapy for pediatric obsessive-compulsive disorder. J Am Acad Child Adolesc Psychiatry. 1998;37(6):663-7. doi: 10.1097/00004583-199806000-00017.

49. Marinova Z, Chuang DM, Fineberg N. Glutamatemodulating drugs as a potential therapeutic strategy in obsessive-compulsive disorder. Curr Neuropharmacol. 2017;15(7):977-95. doi: 10.2174/1570159x1566617032010 4237.

50. Rodriguez CI, Kegeles LS, Levinson A, Feng T, Marcus SM, Vermes D, et al. Randomized controlled crossover trial of ketamine in obsessive-compulsive disorder: proof-ofconcept. Neuropsychopharmacology. 2013;38(12):247583. doi: 10.1038/npp.2013.150.

51. Simpson HB, Shungu DC, Bender J Jr, Mao X, Xu X, Slifstein $M$, et al. Investigation of cortical glutamate-glutamine and $\gamma$-aminobutyric acid in obsessive-compulsive disorder by proton magnetic resonance spectroscopy. Neuropsychopharmacology. 2012;37(12):2684-92. doi: 10.1038/npp.2012.132.

52. Zhang Z, Fan Q, Bai Y, Wang Z, Zhang H, Xiao Z. Brain Gamma-Aminobutyric Acid (GABA) concentration of the prefrontal lobe in unmedicated patients with obsessivecompulsive disorder: a research of magnetic resonance spectroscopy. Shanghai Arch Psychiatry. 2016;28(5):26370. doi: 10.11919/j.issn.1002-0829.216043. 
53. Kapur S, Remington G. Serotonin-dopamine interaction and its relevance to schizophrenia. Am J Psychiatry. 1996;153(4):466-76. doi: 10.1176/ajp.153.4.466.

54. Monckton JE, McCormick DA. Neuromodulatory role of serotonin in the ferret thalamus. J Neurophysiol. 2002;87(4):2124-36. doi: 10.1152/jn.00650.2001.

55. Parent A, Hazrati LN. Functional anatomy of the basal ganglia. I. The cortico-basal ganglia-thalamo-cortical loop. Brain Res Brain Res Rev. 1995;20(1):91-127. doi: 10.1016/0165-0173(94)00007-c.

56. Brennan BP, Tkachenko O, Schwab ZJ, Juelich RJ, Ryan EM, Athey AJ, et al. An examination of rostral anterior cingulate cortex function and neurochemistry in obsessivecompulsive disorder. Neuropsychopharmacology. 2015;40(8):1866-76. doi: 10.1038/npp.2015.36.

57. Viol K, Schiepek G, Kronbichler M, Hartl A, Grafetstätter C, Strasser P, et al. Multi-level assessment of obsessivecompulsive disorder (OCD) reveals relations between neural and neurochemical levels. BMC Psychiatry. 2020;20(1):559. doi: 10.1186/s12888-020-02913-5.

58. Parent A, Hazrati LN. Functional anatomy of the basal ganglia. II. The place of subthalamic nucleus and external pallidum in basal ganglia circuitry. Brain Res Brain Res Rev. 1995;20(1):128-54. doi: 10.1016/0165-0173(94)00008d.

59. Robbins TW, Gillan CM, Smith DG, de Wit S, Ersche KD. Neurocognitive endophenotypes of impulsivity and compulsivity: towards dimensional psychiatry. Trends Cogn Sci. 2012;16(1):81-91. doi: 10.1016/j.tics.2011.11.009.

60. Parmar A, Sarkar S. Neuroimaging studies in obsessive compulsive disorder: a narrative review. Indian J Psychol Med. 2016;38(5):386-94. doi: 10.4103/0253-7176.191395.

61. Abramovitch A, Abramowitz JS, Mittelman A. The neuropsychology of adult obsessive-compulsive disorder: a meta-analysis. Clin Psychol Rev. 2013;33(8):1163-71. doi: 10.1016/j.cpr.2013.09.004.

62. Gillan CM, Morein-Zamir S, Urcelay GP, Sule A, Voon V, Apergis-Schoute AM, et al. Enhanced avoidance habits in obsessive-compulsive disorder. Biol Psychiatry. 2014;75(8):631-8. doi: 10.1016/j.biopsych.2013.02.002.

63. Ahmari SE, Dougherty DD. Dissecting OCD circuits: from animal models to targeted treatments. Depress Anxiety. 2015;32(8):550-62. doi: 10.1002/da.22367.

64. Burguière E, Monteiro P, Feng G, Graybiel AM. Optogenetic stimulation of lateral orbitofronto-striatal pathway suppresses compulsive behaviors. Science. 2013;340(6137):1243-6. doi: 10.1126/science.1232380.

65. Shah DB, Pesiridou A, Baltuch GH, Malone DA, O’Reardon JP. Functional neurosurgery in the treatment of severe obsessive compulsive disorder and major depression: overview of disease circuits and therapeutic targeting for the clinician. Psychiatry (Edgmont). 2008;5(9):24-33.

66. Lopes AC, Greenberg BD, Canteras MM, Batistuzzo MC, Hoexter MQ, Gentil AF, et al. Gamma ventral capsulotomy for obsessive-compulsive disorder: a randomized clinical trial. JAMA Psychiatry. 2014;71(9):1066-76. doi: 10.1001/ jamapsychiatry.2014.1193.

67. Lapidus KA, Stern ER, Berlin HA, Goodman WK. Neuromodulation for obsessive-compulsive disorder. Neurotherapeutics. 2014;11(3):485-95. doi: 10.1007/ s13311-014-0287-9.

68. Trevizol AP, Shiozawa P, Cook IA, Sato IA, Kaku CB, Guimarães FB, et al. Transcranial magnetic stimulation for obsessive-compulsive disorder: an updated systematic review and meta-analysis. J ECT. 2016;32(4):262-6. doi: 10.1097/yct.0000000000000335.

69. Brennan BP, Jacoby RJ, Widge AS. A case of severe intractable contamination-based obsessive-compulsive disorder. JAMA Psychiatry. 2018;75(10):1088-9. doi: 10.1001/jamapsychiatry.2018.0927.

70. Mallet L, Polosan M, Jaafari N, Baup N, Welter ML, Fontaine D, et al. Subthalamic nucleus stimulation in severe obsessive-compulsive disorder. N Engl J Med. 2008;359(20):2121-34. doi: 10.1056/NEJMoa0708514.

71. Luyten L, Hendrickx S, Raymaekers S, Gabriëls L, Nuttin B. Electrical stimulation in the bed nucleus of the stria terminalis alleviates severe obsessive-compulsive disorder. Mol Psychiatry. 2016;21(9):1272-80. doi: 10.1038/ mp.2015.124.

72. Widge AS, Dougherty DD. Deep brain stimulation for treatment-refractory mood and obsessive-compulsive disorders. Curr Behav Neurosci Rep. 2015;2(4):187-97. doi: 10.1007/s40473-015-0049-y.

73. Dougherty DD, Chou T, Corse AK, Arulpragasam AR, Widge AS, Cusin C, et al. Acute deep brain stimulation changes in regional cerebral blood flow in obsessivecompulsive disorder. J Neurosurg. 2016;125(5):1087-93. doi: 10.3171/2015.9.jns151387.

74. Lo MC, Widge AS. Closed-loop neuromodulation systems: next-generation treatments for psychiatric illness. Int Rev Psychiatry. 2017;29(2):191-204. doi: 10.1080/09540261.2017.1282438.

75. Widge AS, Licon E, Zorowitz S, Corse A, Arulpragasam AR, Camprodon JA, et al. Predictors of hypomania during ventral capsule/ventral striatum deep brain stimulation. J Neuropsychiatry Clin Neurosci. 2016;28(1):38-44. doi: 10.1176/appi.neuropsych.15040089.

76. Widge AS, Ellard KK, Paulk AC, Basu I, Yousefi A, Zorowitz $\mathrm{S}$, et al. Treating refractory mental illness with closed-loop brain stimulation: progress towards a patient-specific transdiagnostic approach. Exp Neurol. 2017;287(Pt 4):46172. doi: 10.1016/j.expneurol.2016.07.021. 